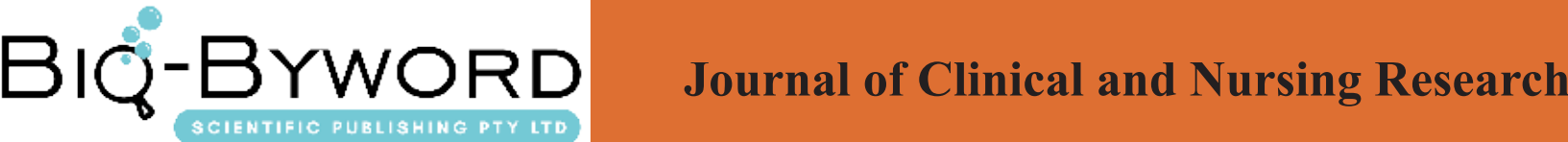

\section{Effect of Humanized Nursing on Emergency Success Rate and Satisfaction in Emergency Nursing}

Kang Yuehua Ling Juanjuan

The First Affiliated Hospital of Northwest University for Nationalities, Yinchuan, Ningxia 750001

\section{ARTICLE INFO}

Article history:

Published online: 15th July, 2017

Key words:

Emergency

humanized nursing

emergency success rate

satisfaction

\section{ABSTRACT}

Objective: To explore the impact of human nursing on emergency success rate and satisfaction in emergency department nursing. Methods: 146 cases of emergency patients were collected from February 2015 to July 2016 in our hospital. The patients were randomly divided into control group and experimental group ( 73 cases). The patients in the control group were treated by routine nursing method. The patients in the experimental group nursing intervention was performed on the basis of humanized care in the control group, and the success rate and satisfaction of the two groups were observed. Results: The success rate of emergency treatment (93.2\%) in the experimental group was significantly higher than that in the control group (79.5\%), the difference was statistically significant $(\mathrm{P}<0.05)$. After the nursing group, the satisfaction rate $91.8 \%$ ) was significantly higher than that of the control group $(65.8 \%)$, which was statistically significant $(\mathrm{P}<0.05)$. Conclusion: Human care in emergency department nursing can effectively improve the success rate of emergency and patient satisfaction.

\section{Introduction}

Emergency department is a more special department in the hospital, the main treatment of critically ill patients, the number of emergency patients, disease, diverse, complex condition ${ }^{[1-2]}$, patients in the diagnosis and treatment process will appear in a variety of unexpected circumstances affect the emergency rescue,

Corresponding author

Ling Juanjuan, Ningxia Yinchuan City is the source of North Street Ningxia Hui Autonomous Region People's Hospital Kang Yuehua. E-mail: 1796100792@163.com 
emergency care is in line with the doctor on the emergency rescue of patients with the work ${ }^{[3]}$, reasonable emergency care can effectively cope with emergency rescue to improve the success rate of emergency treatment. At present, the clinical use of conventional emergency care methods, this method has achieved some results, but because of emergency patients with diverse diseases, complex condition, limited role for some patients. In this study, humanized care was applied to emergency care, and the intervention effect of humanized nursing was analyzed.

\subsection{General information}

Collected from our hospital in February 2015 to July 2016, 146 cases of emergency patients were analyzed, selected criteria ${ }^{[4-5]}$ : (1) All patients are adults over 18 years of age; (2) All patients can communicate properly. The Exclusion criteria ${ }^{[6]}$ : (1) Patients with severe mental retardation; (2) Incomplete data. All patients and their families are informed and agreed to participate in this study. 146 cases of emergency patients were randomly divided into test group and control group of 73 cases, of which 73 patients in the trial group, 38 males and 35 females, aged 20-63 years, mean age $37.3 \pm$ 9.6 years; high school or above 22 Cases, junior high school education in 27 cases, the following education in 24 cases; respiratory disease in 26 cases, 23 cases of cardiovascular disease, trauma in 21 cases. 73 patients in the control group, 39 males and 34 females, aged 2164 years, mean age $38.2 \pm 10.3$ years; high school or higher education in 21 cases, junior high school education in 26 cases, the following educational level 25 cases; respiratory diseases 25 cases, 23 cases of cardiovascular disease, 22 cases of trauma. There was no significant difference between the two groups in the data. There was no significant difference between the two groups $(\mathrm{P}>0.05)$.

\subsection{Methods}

The control group: The patient for routine emergency care, the patient to assess the disease, the symptoms of treatment, to take sedative measures and to guide drug treatment.

Test group: In the control group based on the patient for human care intervention. Details as follows:

1.2.1 For the patient's personal situation for the patient to develop human care program

The status of each emergency patient is different, the patient conducts a comprehensive assessment of the emergency, to understand its incidence, related medical history and mental state, to understand the patient's family background, economic status, education, interpersonal and personality characteristics, In-depth understanding, the establishment of a good relationship between health care, access to patients with full confidence, and on this basis for the development of human care programs for patients. For the patient's incidence, mental state and other factors, timely guidance of patients. The process of full interaction with the patient to carry out human psychological care intervention at the same time, but also timely communication with their families, get more support for the recovery of patients to create a good environment.

1.2.2 For patients with human health knowledge guidance

According to the specific conditions of each patient, education and awareness of the disease, the corresponding patients with the corresponding human health education. The use of easy-to-understand language to explain the relevant knowledge of patients, to provide patients with a reasonable treatment of the disease, prognosis, nursing knowledge, so that patients in a short time to understand the critical knowledge, and actively guide patients with treatment.

1.2.3 To provide patients with personalized psychological care

Emergency patients with emergency symptoms, sudden, often cause great pressure on the patient, the patient's internal tension, anxiety and fear and other factors will have a negative impact on the treatment, so the medical staff need to focus on the patient's personal psychological characteristics of human care intervention to ease the pressure of patients and improve patient compliance with treatment. Nurses in the process of patient care, the need to observe the patient's emotional changes, grasp the patient's mental state, for patients to eliminate doubts at the same time, actively to the patient to convey positive information to help patients build confidence. In addition to the treatment process in patients with their own care intervention, but also need to actively fight for the cooperation of family members, through communication guidance to eliminate the concerns of family members to guide patients with family members to correctly understand and respond to emergencies, to avoid family members due to disagreement caused by differences the normal diagnosis and treatment of patients.

\subsubsection{Humanized counseling and nursing}

Patients in the diagnosis and treatment process, often 
Table 1 Two groups of patients with emergency success rate (n\%)

\begin{tabular}{|c|c|c|c|c|}
\hline Group & $\begin{array}{c}\text { Respiratory } \\
\text { system disease }\end{array}$ & $\begin{array}{c}\text { Cardiovascular } \\
\text { disease }\end{array}$ & Trauma & $\begin{array}{c}\text { Emergency } \\
\text { success rate }\end{array}$ \\
\hline $\begin{array}{c}\text { Control } \\
\text { group }(\mathbf{n}=\mathbf{7 3})\end{array}$ & $20(27.4)$ & $19(26.0)$ & $19(26.0$ & $60(79.5)$ \\
\hline $\begin{array}{c}\text { Test group }(\mathbf{n}=\mathbf{7 3} \\
)\end{array}$ & $25(34.2)$ & $22(30.1)$ & $\begin{array}{c}21(27.4 \\
)\end{array}$ & $68(93.2)$ \\
\hline $\boldsymbol{X}^{2}$ & - & - & - & 4.056 \\
\hline $\mathbf{P}$ & - & - & - & $<0.05$ \\
\hline
\end{tabular}

have doubts need to consult, and some problems often involve personal privacy, or some patients because of their own personal psychological needs is not easy through regular counseling channels for consultation. For patients to establish a special way of human services, for different patients to provide personalized counseling services, timely and effective for patients to help, care process to fully respect the patient to do privacy work.

\subsubsection{Humanized Environmental Care}

For the patient>s ward environment In addition to cleaning and timely ventilation and other normal links, the nursing staff through the communication with the patient, to understand the patient's personal preferences, in the environment and the placement of items and other aspects of the views of patients seeking to create a good treatment environment. A good environmental atmosphere helps the patient's nervousness and anxiety. Arrange special staff to maintain environmental conditions and maintain good environmental temperature and humidity.

\subsection{Observe indicators}

1.3.1 Emergency rescue success criteria
Patients with emergency rescue after the end of the vital signs of patients with relief or disappearance, in the hospital or transferred to hospital without life risk. 1.3.2 Satisfaction evaluation

Using the satisfaction table to assess, are divided into very satisfied, satisfied, average, dissatisfied and very dissatisfied with the 5 to evaluate the questionnaire to conduct a survey. Satisfaction $=$ Very Satisfactory Rate + Satisfaction Rate.

\subsection{Statistical methods}

Data analysis using SPSS23.0 statistical software, data consistent with the normal distribution, measurement data with the mean \pm standard deviation $(\bar{x} \pm s)$ said, the measurement data for $t$ test, counting the number of cases and $\%$, counting data for $\mathrm{x}^{2}$ test, $\mathrm{P}<0.05$ indicates a difference, statistically significant.

\section{Results}

2.1 Comparison of emergency success rate between the two groups of patients

Table 1 shows that the emergency group success rate $(93.2 \%)$ was significantly higher than the control group

Table 2 Comparison of satisfaction in both groups ( $\mathrm{n} \%)$

\begin{tabular}{|c|c|c|c|c|c|c|}
\hline Group & $\begin{array}{c}\text { Very satis- } \\
\text { fied }\end{array}$ & Satisfactory & Average & Dissatisfied & $\begin{array}{c}\text { Very dissat- } \\
\text { isfied }\end{array}$ & Satisfaction \\
\hline $\begin{array}{c}\text { Control } \\
\text { group (n=73 } \\
)\end{array}$ & $23(31.5)$ & $25(34.2)$ & $18(24.7)$ & $5(6.8)$ & $2(2.7)$ & $48(65.8)$ \\
\hline $\begin{array}{c}\text { Test } \\
\text { group (n=73 } \\
)\end{array}$ & $36(49.3)$ & $31(42.5)$ & $5(6.8)$ & $1(1.4)$ & $0(0.0)$ & $67(91.8) *$ \\
\hline$X^{2}$ & - & - & - & - & - & 6.295 \\
\hline
\end{tabular}


(79.5\%), a statistically significant $(\mathrm{P}<0.05)$. See Table 1.

2.2 Comparison of two groups of patient's satisfaction rate

Table 2 shows that the satisfaction of the test group $(91.8 \%)$ was significantly higher than the control group $(65.8 \%)$, with statistical significance $(\mathrm{P}<0.05)$. See Table 2 .

\section{Discussion}

Emergency patients in patients with complex condition, the symptoms are critical, patients and their families in the emergency situation will have a lot of pressure ${ }^{[7-}$ ${ }^{8]}$, while the emergency department for many patients, medical staff in the diagnosis and treatment process will also face a lot of problems such as emergency patients may panic, anxiety and other phenomena affect the diagnosis and treatment, the patient's family may also panic and affect the order of the scene. In the process of diagnosis and treatment of patients, emergency care is a very important intervention measures, reasonable emergency care can be a good doctor with the treatment for the diagnosis and treatment of patients with a strong guarantee. Routine emergency care that is often used in clinical practice has played a certain role in the emergency process of patients, but because of the complex and diverse condition of emergency patients, the use of conventional methods in many cases is poor. Human care is a patient-centered way of care, this way according to the incidence of different emergency patients, their own characteristics and other comprehensive assessment, and through the patient and their families to fully understand the basis of a flexible patient care intervention. Humanity care has a higher demand for health care workers themselves. In the course of nursing care for patients, some urgent problems need to be dealt with. In the face of complex and sudden problems, the nurses need timely and accurate judgment and timely treatment. Due to delay caused by the adverse consequences of the production. In the care of patients, the patient's psychological care is also an important aspect, timely relief of anxiety and anxiety in patients with mental state, improve patient compliance can help emergency treatment.

Humanized care to the patient as a starting point, to better understand the patient and service, in the nursing intervention process due to human and should be very good in line with the condition of different patients ${ }^{[9-10]}$. Studies have shown that human care in the emergency care has a very good care effect, can improve the suc- cess rate of emergency rescue. Du Peihua ${ }^{[11]}$ and other stated that in the application of human care in emergency patients in the study found that human care in emergency care in the effective improvement of patient care quality and patient satisfaction, but also inspired the creativity of nurses The This method is not only conducive to the diagnosis and treatment of patients, but also improve the emergency department's own service level. Long Xiao-yu ${ }^{[12]}$ and other stated that in the human care services in the emergency department of the application of the results of the study found that human care in emergency care in the effective improvement of the quality of care and rescue success rate. In this study also found that human care is very good to adapt to different situations of emergency patients, not only effectively improve the success rate of emergency rescue patients, but also by the majority of patients and their families to improve the recognition of satisfaction.

In summary, emergency nursing care in human care can effectively improve the success rate of emergency and patient satisfaction, it is worth promoting the application.

\section{References:}

[1] Guan Haiying. Maternity Hospital Emergency Department of Human Nursing Care for the Satisfaction of Patients [J]. China Health Industry, 2016, 13 (9): 49-51.

[2] Huang Rongzhen. Emergency Nursing Nurses on the Effectiveness of Human Management [J]. Contemporary Medicine, 2015, 15 (12): 109-110.

[3] Jiang Chun. Human Health Care on the Impact of Emergency Nursing Satisfaction Analysis [J] .Group primary medicine, 2016, 20 (4): 555-556.

[4] Liu Ying, Zhao Ting. Humanized Nursing In Acute Myocardial Infarction In Patients With Emergency Nursing Before Use [J]. Qilu Nursing Journal, 2014, 20 (1): 13-15.

[5] Lai Yuming. Emergency department to carry out quality care services practice experience [J]. China Disability Medicine, 2012, 20 (6): 132-133.

[6] Yang Haoqi, Zheng Xiaoli. The Whole Nursing Model in the Emergency Treatment of Critically Ill Patients in the Application [J]. Chinese Journal of Traditional Chinese Medicine, 2016, 24 (3): 72-74.

[7] Wu Dan, Lan Rongli, Fu Wenjuan. Humanized Care For Emergency Care In Patients With Observation [J]. Contemporary Medicine, 2016, 22 (22): 114-115. 
[8] Wang Aiying, Liu Hailing, Chen Lihong. Humanistic Care for the Emergency Department to Create Quality Care Services [J]. Jilin Medical, 2013, 34 (20): 4140-4142.

[9] Zhu Haiyan. Effect of Humanized Nursing on Anxiety of Outpatient Fever Patients [J]. Journal of Medical Theory and Practice, 2014, 27 (5): 691-693.

[10] Zhao Yuhong, Liu Shuangjun, Zhang Ying, et al. Humanized Care in Elderly Patients in the Process of
Infusion [J]. Journal of Modern Medicine, 2015, 22 (23): 10-11.

[11] Du Peihua, Zhao Wenfeng, Hu Xiangmei. Application of Humanized Nursing in Emergency Patients [J]. Laboratory Medicine and Clinical Medicine, 2016, 13 (12): 1720-1721.

[12] Long Xiao Yu. Human Care Services in the Emergency Department of the Application of the Results [J]. Clinical Medicine Engineering, 2015, 22 (5): 636-637. 